\title{
Electron Optics.*
}

\section{By Prof. G. P. Thomson, F.R.S.}

$\mathrm{A}^{\mathrm{LM}}$ all progress in physics has come from comparing the unfamiliar with the familiar. One could scarcely have a better example than the story of Newton's apple-for this comparison assimilated the motion of the moon to the motion of a falling body. The great essential is to use an idea with which one is already familiar. It is less important that the idea should be really understood-if, indeed, anything in the physical world ever can be understood in any absolute sense. The falling of the apple was not understood then (one may perhaps doubt if we understand it much better now, when we say that it is a consequence of the curvature of space), but it was at least familiar, and the mind needs familiar images with which to work.

Now, in the study of the very small there are two pictures which have proved their use. One is as old as the Greeks : the idea of a particle, hard, small, smooth, an idealised grain of sand. Generally it is pictured as moving, and the universe as an endless game of snooker with every ball played at once. The other view is only seven years old, but it is a promising youngster and in the last few years has come to take an equal place with its elder brother, so producing a curious duality in our views of matter. I refer to the idea of matter as waves, which was invented by Louis de Broglie. Now, light has long been regarded as waves, and to many physicists, at least, waves of light are more familiar in their properties than the more tangible waves of water or of sound. This analogy between matter and light is a good one, but, like all analogies, there are points at which it fails.

According to de Broglie, any piece of matter should have wave properties, but in most cases the wave-length would be too small to detect, even with the most refined experiments. The heavier the piece of matter and the faster it is moving, the shorter is the wave-length, and although recently the wave properties of atoms as a whole have been detected, most of the experimental work has been done with the lightest known pieces of matter, namely, electrons. Even with these, the wavelength is usually much less than that of light. Thus for the electrons which form part of a beam of cathode rays from a discharge in rarefied air, the wave-length is usually about one fortieth of the size of an average atom, and only about one hundredthousandth of the wave-length of red light. Actually, if the voltage is 30,000 volts, the wave-length is about $7 \times 10^{-10} \mathrm{~cm}$.

The characteristic feature of those waves which one can actually see and feel is the periodic motion of the water or other medium, but when one comes to waves in which one can no longer actually see the medium bobbing up and down, the most characteristic wave property is that called 'interference'. This is the property by which, for example, two beams of light can produce darkness, and is explained at once as due to the superposition of two

- Friday evening discourse delivered at the Royal Institution on Dec. 4. No. 3246, VoL. 129] trains of waves in such a way that the crest of one wave neutralises the trough of another.

In the case of light, this leads to a pattern of light and dark bands, the sharpness of which may be much increased by using not two waves only but a large number of wavelets produced by the scattering of a single wave from a number of obstacles arranged in a regular order. To get a satisfactory pattern, the distance apart of the scattering objects must not be too great compared with the wavelength of the light. This led Laue to use the atoms of a crystal as scattering centres to measure the very short wave-length of $\mathrm{X}$-rays. The use of a crystal, however, introduces a complication, for we are not only concerned with the wavelets from one plane of atoms but also with those from successive planes. The result is that only if the crystal is correctly adjusted to the incident beam will there be a strong beam selected by interference. If the crystal is powdered and the pieces are arranged at random in the path of the rays, there will always be some pieces correctly adjusted, and the beams from them will lie on cones and their intersection with a screen will show as rings. The absolute sizes of the rings are proportional to the wave-length and inversely as the spacing of the atoms, while the relative sizes of the different rings in any one experiment depend on the arrangement of the atoms in the crystal.

Now, electrons are certainly scattered by atoms. If they are like waves, they should give interference and the scattered waves should be concentrated in certain directions; presumably the electrons will show themselves most where the waves are concentrated. In my early experiments, this was done by passing a narrow beam of cathode rays through a thin foil and receiving the scattered rays on a photographic plate. When developed, these plates showed ring patterns which were in agreement with those calculated from de Broglie's theory and the known crystal structure of the metal films used. To get good results the films had to be of the order of $10^{-6} \mathrm{~cm}$., and this limits the substances with which one can work. I have found, however, that equally good results can be got by reflecting the rays from a block of material, the face of which has been suitably prepared. It is probable that the rays pass through minute projections on the surface, so that the reflection is only apparent. In this way ring patterns have been obtained with a variety of substances, such, for example, as spluttered metals and metals which have undergone surface oxidation or other chemical change. The method enables one to examine a very thin layer of the substance, for the effective depth of penetration is of the order $10^{-6} \mathrm{~cm}$. It appears that almost any surface will give some kind of a pattern, providing that it is reasonably flat.

The concentration due to interference is, as we have seen, much more marked if there are many scattering centres arranged in order. This is so in 
the case of the above experiments; for though the crystals are small enough to be very numerous, even in a small piece of material, they each contain a very large number of atoms. Recently, Wierl has made experiments of the same character with gases. Here the scattering particles are the atoms, of which there are only a few in each molecule; so though rings are formed, they are not very sharp. Nevertheless, the theory holds, and Wierl has calculated the distance apart of the atoms in various molecules from the sizes of the rings. He has been able to draw a number of interesting conclusions about the relative positions of the atoms in the molecules. For example, he finds that the carbon atoms in the benzene rings lie in a plane. Mr. French has recently found similar diffuse rings from the polished surfaces of metals. These rings appear to be due to the noncrystalline Beilby layer.

The apparatus I have described will also serve for single crystals. Here, however, we should expect nothing unless the crystal were suitably adjusted, and then only a small number of spots. With a cleaved surface of rock salt these expectations are approximately fulfilled, though there is enough latitude from various causes for some sort of a spot to appear for almost any setting of the crystal. In addition, there appear a number of black and white lines, which have been ably explained by the discoverer, Kikuchi, as due to double scattering.

Some experiments with single crystals of metals have led to rather surprising results. When cut so that the face is crystallographically a simple one, these crystals give an extended pattern of spots of a very regular character. The pattern is just what we expect from a single layer of atoms arranged in the crystal form and set normal to the rays. The agreement is exact and extends to small details. The pattern is the polar reciprocal of the atomic arrangement known to exist in the crystal, each spot of the pattern corresponds to a line of atoms in the crystal, and the whole plane of atoms behaves like two crossed optical gratings. Clearly, however, a single plane of atoms sticking out at right angles to the main surface is a mechanical impossibility. The surface was actually prepared by etching and was probably covered with pits and pyramids, through which the electrons pass. The reason for the extended pattern is that the thickness in the path of the rays is so small that the successive layers of atoms are not badly out of step over a considerable range of angle. This approximate agreement is helped by the small angle through which the rays are turned out.

Some recent work in conjunction with $\mathrm{Mr}$. Stuart on spluttered platinum films has shown that while the usual structure is that of normal platinum, abnormal types sometimes occur. One of these gives a pattern of straight lines, which is unstable and disappears under the action of the rays. It appears to be due to the presence on the surface of small pieces of some crystalline structure in which the atoms are arranged accurately in layers parallel to the surface but each separate layer is largely chaotic. We have not been able to determine whether the atoms are those of platinum or of a gas. Another abnormal structure shows the presence of extra rings, which may be associated with catalytic activity of the platinum.

While these experiments show the close resemblance between the behaviour of the electrons and that of light in those experiments which were the original proof of its wave character, yet, all the same, one must not entirely abandon the particle idea. When the electron reaches the photographic plate, it seems to forget that it is a wave and becomes a particle. If the plate were examined under a high magnification, the pattern would show up as a number of black specks, each corresponding to one

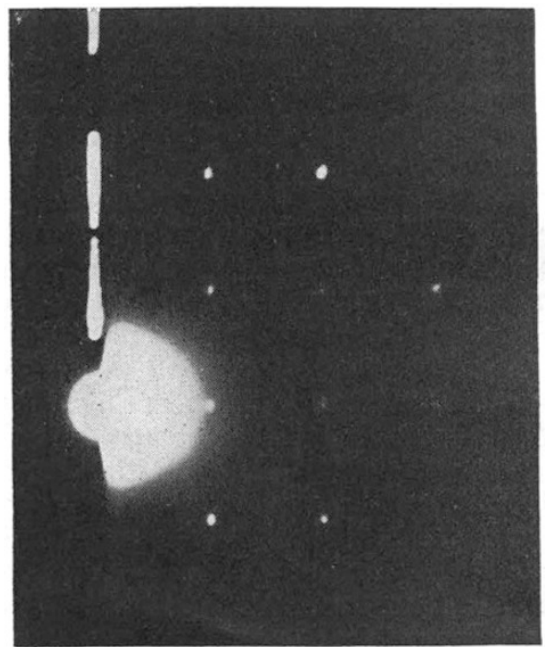

FIG. 1.-Diffraction pattern formed by electrons reflected from a cube face of a silver crystal. The interrupted line was added afterwards.

grain of silver bromide made developable. Each electron affects one grain only. Yet the waves which interfere must have been scattered from atoms over a wide front, and the sharpness of the ring and spots shows that there has been a long train of waves acting, just as a sharp line in an optical spectrum implies a long train of coherent waves. On the wave side of its nature the electron is a widely extended entity which in a sense occupies the whole region in which it might be found. An electron is like an able guerilla leader who occupies a wide area with rumours of his presence, but when he strikes, he strikes with his whole force. No analogy is perfect, and though the analogy between light and electrons even extends to this curious duality of wave and particle, it breaks down in the end.

Electrons are essentially different from light. They are acted on by electric and magnetic forces, and react on the bodies which cause these forces. Light does not: it only affects matter when it actually hits it; there is no action at a distance. Further, the electronic waves are a trifle less real than those of light. No one, it is true, has ever actually observed the frequency of visible light, but that of very long wireless waves can be directly followed, and the difference must be only one of degree. Now, so far as we know at present, the frequency of electron waves is quite unobservable. It is always the wave-length which we find. Possibly we could observe differences of frequency under 
very special conditions, but the absolute values seem to have no significance except as mathematical symbols. Different values can be assigned to this frequency, and the calculated results still agree with experiment.

Again, the fluctuating quantities or 'displacements' in the light wave have a definite physical meaning, namely, electric and magnetic intensities. No one has observed the corresponding quantity in the electronic wave. Indeed, in the most suc- cessful forms of the theory it is not even a real number. Only the intensity of the wave has a physical meaning, namely, the chance that an electron will appear at the place in question. The two views of electrons, as particle and as wave, are parables, each enshrining a part of the truth. Whether between them they include all that we need to know, as some physicists believe, or whether there are not other aspects, still undreamed of, is for the future to decide.

\section{Dental Caries and Diet.}

$\mathrm{T}$ HE researches of Mrs. Mellanby on the part played by diet in the development of the teeth and jaws in animals have already been referred to in these columns. ${ }^{1}$ A report on the relationship between diet and the structure of human teeth is in course of preparation. Mrs. Mellanby's work has also led to the conclusion that it is possible to influence the structure, and thereby the resistance to disease, of teeth after eruption. It was obviously desirable that this conclusion should be confirmed by a large-scale clinical trial. A preliminary report of such a trial has now been issued by the Committee upon Dental Disease. ${ }^{2}$

The investigation was carried out at three residential institutions for children near Birmingham, under the control of the poor law and later the local education authority. The teeth of the children were examined every six months by $\mathrm{Mr}$. A. Deverall: the diets were controlled by Miss E. Brinton and Miss M. Reynolds. The number of children included in the investigation was 332 , aged from 5 years to 14 years : the duration of the test was two years. The children were divided into four groups, all of which were on a similar and generous basal diet. At the first institution 1-1.5 oz. treacle daily was added to the diet; at the second 1-1.5 tablespoonfuls (14-21 c.e.) daily of olive oil to that of one group and a similar amount of olive oil containing radiostol (irradiated ergosterol) to that of the remaining children; at the third institution the addition to the diet was $14-21$ c.c. cod-liver oil daily.

The dental examination made at each inspection included the number and structure of the fully erupted teeth, the condition as regards caries, of which three degrees were arbitrarily recognised, and the condition of the gums. By using a numerical notation for the degree of caries, it was possible to obtain the total caries figure for any group of children, and also the average caries figure by dividing the total by the number of teeth in the group. All teeth lost subsequent to the first inspection continued to be counted in later inspections, since otherwise the extraction of a carious tooth would appear to lead to a sudden improvement in the condition as regards caries. The incidence of caries in a group was shown by the number of carious teeth: the extent of caries by the average caries figure. The latter could only show a decrease when the number of teeth in the group increased by the eruption of fresh teeth, which were not attacked, or only slightly, by caries, since the total caries figure could not decrease with the passage of time.

The investigation reported was actually carried out in two parts : in the first the effects of adding treacle, olive oil, or cod-liver oil to the diet were compared; in the second the effects of olive oil and radiostol. The olive oil group was to a large extent the same in each investigation. Each group consisted of 65-85 children. The results obtained were briefly as follows: the increase in the percentage of carious teeth in the group given treacle was 10 , in those given olive oil 8 , and cod-liver oil 3 , in the first experiment; in the second the olive oil group showed a percentage increase of 7 and the radiostol group one of 2 . The percentage increases of the average caries figures for the five groups were respectively $42,46,10,31$, and 10. Statistically, all the differences are significant except that between the average caries figures in the second experiment. Further analysis of the figures indicated that similar differences were observed when the deciduous teeth, the permanent teeth or the first and second premolars (upper and lower), or first permanent molars (upper and lower), were considered separately. In the first investigation the actual number of carious teeth approximately doubled at the institutions in which treacle and olive oil were the dietary supplements, but only increased 50 per cent when cod-liver oil was given. In the second investigation the number of carious teeth per child rose from 4.5 to 6.2 in the radiostol group and from 3.8 to 6.4 in the control group; for the permanent teeth the figures were $1 \cdot 8-3 \cdot 1$ and $1 \cdot 6-3 \cdot 7$ respectively. Very similar figures were obtained in the first experiment.

Considering the two investigations together, it was found that the increase of the average caries figure of the permanent teeth in the two vitamin groups was about two-fifths of that in the control groups. In general, the results agreed closely with those previously obtained at Sheffield with a smaller number of younger children suffering from surgical tuberculosis and showing a higher initial incidence of caries.

It appears, therefore, that the addition of vitamin $\mathrm{D}$ to a standard dietary will reduce the increase in the incidence and extent of caries, which occurs with the passage of time, by about two-thirds.

1 NATURe, vol. 125 , p. $604 ; 1930$ : vol. 127, p. $977 ; 1931$

2 "The Influence of Diet on Caries in Children's 'Teeth" (Interim Report). By the Committee upon Dental Disease. Medical Research Council. Special Report Series No. 159. (London: H.M. Stationery Office, 1931.) bd. net.

$$
\text { No. 3246, VoL. 129] }
$$

